\title{
Cosmological bounds on neutrino degeneracy improved by flavor oscillations
}

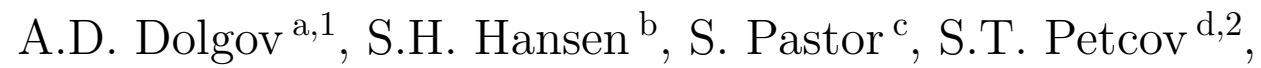 \\ G.G. Raffelt ${ }^{c}$, D.V. Semikoz ${ }^{c, 3}$ \\ ${ }^{a}$ INFN section of Ferrara, Via del Paradiso 12, 44100 Ferrara, Italy \\ ${ }^{\mathrm{b}}$ NAPL, University of Oxford, Keble road, OX1 3RH, Oxford, UK \\ ${ }^{\mathrm{c}}$ Max-Planck-Institut für Physik, Föhringer Ring 6, 80805 München, Germany \\ d Scuola Internazionale Superiore di Studi Avanzati and INFN section of Trieste, \\ Via Beirut 2-4, 34014 Trieste, Italy
}

\begin{abstract}
We study three-flavor neutrino oscillations in the early universe in the presence of neutrino chemical potentials. We take into account all effects from the background medium, i.e. collisional damping, the refractive effects from charged leptons, and in particular neutrino self-interactions that synchronize the neutrino oscillations. We find that effective flavor equilibrium between all active neutrino species is established well before the big-bang nucleosynthesis (BBN) epoch if the neutrino oscillation parameters are in the range indicated by the atmospheric neutrino data and by the large mixing angle (LMA) MSW solution of the solar neutrino problem. For the other solutions of the solar neutrino problem, partial flavor equilibrium may be achieved if the angle $\theta_{13}$ is close to the experimental limit $\tan ^{2} \theta_{13} \lesssim 0.065$. In the LMA case, the BBN limit on the $\nu_{e}$ degeneracy parameter, $\left|\xi_{\nu}\right| \lesssim 0.07$, now applies to all flavors. Therefore, a putative extra cosmic radiation contribution from degenerate neutrinos is limited to such low values that it is neither observable in the large-scale structure of the universe nor in the anisotropies of the cosmic microwave background radiation. Existing limits and possible future measurements, for example in KATRIN, of the absolute neutrino mass scale will provide unambiguous information on the cosmic neutrino mass density, essentially free of the uncertainty of the neutrino chemical potentials.
\end{abstract}

Key words: Physics of the Early Universe; Neutrino Physics 


\section{Introduction}

The cosmic matter and radiation inventory is known with ever increasing precision, but many important questions remain open. The cosmic neutrino background is a generic feature of the standard hot big bang model, and its presence is indirectly established by the accurate agreement between the calculated and observed primordial light-element abundances. However, the exact neutrino number density is not known as it depends on the unknown chemical potentials for the three flavors. (In addition there could be a population of sterile neutrinos, a hypothesis that we will not discuss here.) The standard assumption is that the asymmetry between neutrinos and anti-neutrinos is of order the baryon asymmetry $\eta \equiv\left(n_{B}-n_{\bar{B}}\right) / n_{\gamma} \simeq 6 \times 10^{-10}$. This would be the case, for example, if $B-L=0$ where $B$ and $L$ are the cosmic baryon and lepton asymmetries, respectively. While $B-L=0$ is motivated by scenarios where the baryon asymmetry is obtained via leptogenesis [1], there are models for producing large $L$ and small $B$ [2-5].

In order to quantify a putative neutrino asymmetry we assume that well before thermal neutrino decoupling a given flavor is characterized by a Fermi-Dirac distribution with a chemical potential $\mu_{\nu}, f_{\nu}(p, T)=\left[\exp \left(E_{p} / T-\xi_{\nu}\right)+1\right]^{-1}$, where $\xi_{\nu} \equiv \mu_{\nu} / T$ is the degeneracy parameter and $E_{p} \simeq p$ since we may neglect small neutrino mass effects on the distribution function. For anti-neutrinos the distribution function is given by $\xi_{\bar{\nu}}=-\xi_{\nu}$.

A neutrino chemical potential modifies the outcome of primordial nucleosynthesis in two different ways [6]. The first effect appears only in the electron sector because electron neutrinos participate in the beta processes which determine the primordial neutron-to-proton ratio so that $n / p \propto \exp \left(-\xi_{e}\right) .{ }^{4}$ Therefore, a positive $\xi_{e}$ decreases $Y_{\mathrm{p}}$, the primordial ${ }^{4} \mathrm{He}$ mass fraction, while a negative $\xi_{e}$ increases it, leading to an allowed range

$$
-0.01<\xi_{e}<0.07
$$

compatible with $\xi_{e}=0$ (see Refs. [7-10] and Sec. 5). A second effect is an increase of the neutrino energy density for any non-zero $\xi$ which in turn increases the expansion rate of the universe, thus enhancing $Y_{\mathrm{p}}$. This applies to

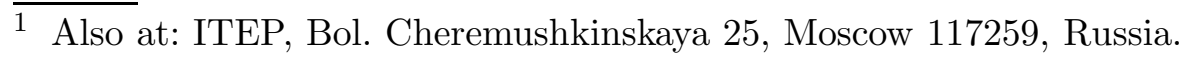

2 Also at: Institute of Nuclear Research and Nuclear Energy, Bulgarian Academy of Sciences, 1784 Sofia, Bulgaria.

3 Also at: Institute for Nuclear Research of the Academy of Sciences of Russia, 60th October Anniversary Prospect 7a, Moscow 117312, Russia.

4 We use the notation $\xi_{e} \equiv \xi_{\nu_{e}}$ etc. to avoid double subscripts. We never discuss charged-lepton chemical potentials so that there should be no confusion. 
all flavors so that the effect of chemical potentials in the $\nu_{\mu}$ or $\nu_{\tau}$ sector can be compensated by a positive $\xi_{e}$. Altogether the big-bang nucleosynthesis (BBN) limits on the neutrino chemical potentials are thus not very restrictive.

Another consequence of the extra radiation density in degenerate neutrinos is that it postpones the epoch of matter-radiation equality. In the cosmic microwave background radiation (CMBR) it boosts the amplitude of the first acoustic peak of the angular power spectrum and shifts all peaks to smaller scales. Moreover, the power spectrum of density fluctuations on small scales is suppressed [11,12], leading to observable effects in the cosmic large-scale structure (LSS).

A recent analysis of the combined effect of a non-zero neutrino asymmetry on BBN and CMBR/LSS yields the allowed regions [13]

$$
-0.01<\xi_{e}<0.22, \quad\left|\xi_{\mu, \tau}\right|<2.6,
$$

in agreement with similar bounds in $[14,15]$. These limits allow for a very significant radiation contribution of degenerate neutrinos, leading many authors to discuss the implications of a large neutrino asymmetry in different physical situations. These include the explanation of the former discrepancy between the BBN and CMBR results on the baryon asymmetry [16] or the origin of the cosmic rays with energies in excess of the Greisen-Zatsepin-Kuzmin cutoff [17]. In addition, if the present relic neutrino background is strongly degenerate, it would enhance the contribution of massive neutrinos to the total energy density $[18,19]$ and affect the flavor oscillations of the high-energy neutrinos [20] which are thought to be produced in the astrophysical accelerators of high-energy cosmic rays.

The limits in Eq. (2) ignore neutrino flavor oscillations, an assumption which is no longer justified in view of the experimental signatures for neutrino oscillations by solar and atmospheric neutrinos. For zero initial neutrino chemical potentials, the flavor neutrinos have the same spectra so that oscillations produce no effect. This is true up to a small spectral distortion caused by the heating of neutrinos from $e^{+} e^{-}$annihilations, an effect which is different for electron and muon/tau neutrinos and which causes a small relative change in the final production of ${ }^{4} \mathrm{He}$ of order $10^{-3}$ [21]. This relative change is slightly enhanced by neutrino flavor oscillations $[22,23]$. In the presence of neutrino asymmetries, flavor oscillations equalize the neutrino chemical potentials if there is enough time for this relaxation process to be effective [24]. If flavor equilibrium is reached before $\mathrm{BBN}$, then the restrictive limits on $\xi_{e}$ in Eq. (1) will apply to all flavors, in turn implying that the cosmic neutrino radiation density is close to its standard value. As a consequence, it is no longer necessary to use the neutrino radiation density as a fit parameter for CMBR/LSS analyses, unless one considers exotic models with decaying massive particles. 
The effects of flavor oscillations on possible neutrino degeneracies have been considered in [20], where it was concluded that flavor equilibrium was achieved before the BBN epoch if the solar neutrino problem was explained by the largemixing angle (LMA) solution. The LMA solution is favored by the current solar neutrino data. Thus, it was concluded that in the LMA case a large cosmic neutrino degeneracy was no longer allowed.

We revisit this problem because the flavor evolution of the neutrino ensemble is more subtle than previously envisaged if medium effects are systematically included. Contrary to the treatment of Ref. [20], the refractive effect of charged leptons can not be ignored, and actually is one of the dominant effects. While the background neutrinos produce an even larger refractive term, its effect is to synchronize the neutrino oscillations $[25,26]$ which remain sensitive to the charged-lepton contribution. Still, equilibrium is essentially, but not completely, achieved in the LMA case so that our final conclusion is qualitatively similar to that of Ref. [20].

One counter-intuitive subtlety is that the neutrino self-potential actually can suppress oscillations in a situation where the excess of neutrinos in one flavor is exactly matched by an excess of anti-neutrinos in another flavor. In this case the synchronized oscillation frequency is zero so that oscillations begin only once the cosmic expansion has diluted the self-term. Therefore, one can construct cases where flavor equilibrium is not achieved before BBN even in the LMA case. However, this is only possible for specially chosen initial conditions where $\left|\xi_{\nu}\right|$ is equal for all flavors, but the absolute signs may be different. For the purpose of deriving limits on $\left|\xi_{\nu}\right|$, however, this case is equivalent to the one where equilibrium is achieved, the only important point being that $\left|\xi_{\nu}\right|$ is approximately equal for all flavors at the BBN epoch.

Another subtlety appears in a full three-flavor analysis. We show that achieving equilibrium in the LMA case does not depend on the value of the mixing angle $\theta_{13}$, to which strict limits from reactor experiments apply. Moreover, for the non-LMA solutions of the solar neutrino problem, partial flavor equilibrium may be reached if the angle $\theta_{13}$ is small but non-zero.

In Sec. 2 we set up the formalism to study primordial flavor oscillations. We then turn in Sec. 3 to the primordial flavor evolution of a simplified system where $\nu_{e}$ mixes maximally with one other flavor. This two-flavor case will illustrate many of the important subtleties of our problem. Then we turn in Sec. 4 to realistic three-flavor situations which involve yet further complications. In Sec. 5 we finally derive new limits on the degeneracy parameters and summarize our findings. 


\section{Neutrino flavor oscillations in the early universe}

In order to study neutrino oscillations in the early universe we characterize the neutrino ensemble in the usual way by generalized occupation numbers, i.e. by $3 \times 3$ density matrices for neutrinos and anti-neutrinos as described in $[27,28]$. The form of the density matrices for a mode with momentum $p$ is

$$
\rho(p, t)=\left(\begin{array}{ccc}
\rho_{e e} & \rho_{e \mu} & \rho_{e \tau} \\
\rho_{\mu e} & \rho_{\mu \mu} & \rho_{\mu \tau} \\
\rho_{\tau e} & \rho_{\tau \mu} & \rho_{\tau \tau}
\end{array}\right), \quad \bar{\rho}(p, t)=\left(\begin{array}{ccc}
\bar{\rho}_{e e} & \bar{\rho}_{\mu e} & \bar{\rho}_{\tau e} \\
\bar{\rho}_{e \mu} & \bar{\rho}_{\mu \mu} & \bar{\rho}_{\tau \mu} \\
\bar{\rho}_{e \tau} & \bar{\rho}_{\mu \tau} & \bar{\rho}_{\tau \tau}
\end{array}\right)
$$

where overbarred quantities refer to anti-neutrinos. The diagonal elements of the density matrices correspond to the usual occupation numbers of the different flavors. The definition of the density matrix for anti-neutrinos is "transposed" relative to that for neutrinos, allowing one to write the equations of motion in a compact form [27].

The equations of motion for the density matrices relevant for our situation of interest are $[27,29]$

$$
\begin{aligned}
& i \partial_{t} \rho_{p}=+\left[\frac{M^{2}}{2 p}, \rho_{p}\right]+\sqrt{2} G_{\mathrm{F}}\left[\left(-\frac{8 p}{3 m_{\mathrm{W}}^{2}} E+\rho-\bar{\rho}\right), \rho_{p}\right]+C\left[\rho_{p}\right], \\
& i \partial_{t} \bar{\rho}_{p}=-\left[\frac{M^{2}}{2 p}, \bar{\rho}_{p}\right]+\sqrt{2} G_{\mathrm{F}}\left[\left(+\frac{8 p}{3 m_{\mathrm{W}}^{2}} E+\rho-\bar{\rho}\right), \bar{\rho}_{p}\right]+C\left[\bar{\rho}_{p}\right]
\end{aligned}
$$

where we use the notation $\rho_{p}=\rho(p, t)$ and $[\cdot, \cdot]$ is the usual commutator. Further, $M^{2}$ is the mass-squared matrix in the flavor basis; in the mass basis it would be $\operatorname{diag}\left(m_{1}^{2}, m_{2}^{2}, m_{3}^{2}\right)$. The diagonal matrix $E$ represents the energy densities of charged leptons. For example, $E_{e e}$ is the energy density of electrons plus that of positrons. The density matrix $\rho$ is the integrated neutrino density matrix so that, for example, $\rho_{e e}$ is the $\nu_{e}$ number density while $\bar{\rho}_{e e}$ is the $\bar{\nu}_{e}$ number density. The term proportional to $(\rho-\bar{\rho})$ is non-linear in the neutrino density matrix and represents self-interactions. Finally, $C[\cdot]$ is the collision term which is proportional to $G_{\mathrm{F}}^{2}$. We have neglected a refractive term proportional to the neutrino energy density which is much smaller than the $(\rho-\bar{\rho})$ term in our present situation where the neutrino asymmetries are assumed to be large. On the other hand, we have neglected the usual term which is proportional to the charged-lepton asymmetries. This asymmetric term is always negligible; at early times (high temperatures) it is negligible compared to the $E$ term, while at temperatures near $n / p$ freeze out $(T \simeq 1 \mathrm{MeV})$ it is negligible compared to the vacuum term $M^{2} / 2 p$ if the mass-squared differences coincide with those characterizing the atmospheric neutrino and the solar neutrino LMA oscillations. 
In the expanding universe we need to substitute $\partial_{t} \rightarrow \partial_{t}-H p \partial_{p}$ with $H$ the cosmic expansion parameter. We have taken this into account rewriting the equations in terms of comoving variables, as listed in the Appendix. We solve these equations numerically, calculating the evolution of $\rho$ and $\bar{\rho}$ on a grid of neutrino momenta.

\section{Two-flavor oscillations}

\subsection{Equations of motion for the polarization vectors}

In order to develop a first understanding of flavor oscillation in the early universe it will be useful to study first a two-flavor situation involving $\nu_{e}$ and $\nu_{\mu}$. The usual relation between flavor and mass eigenstates is

$$
\begin{aligned}
& \nu_{e}=\cos \theta \nu_{1}+\sin \theta \nu_{2}, \\
& \nu_{\mu}=-\sin \theta \nu_{1}+\cos \theta \nu_{2} .
\end{aligned}
$$

The $2 \times 2$ density matrices are

$$
\begin{aligned}
& \rho(p, t)=\left(\begin{array}{ll}
\rho_{e e} & \rho_{e \mu} \\
\rho_{\mu e} & \rho_{\mu \mu}
\end{array}\right)=\frac{1}{2}\left[P_{0}(p, t)+\boldsymbol{\sigma} \cdot \mathbf{P}(p, t)\right] \\
& \bar{\rho}(p, t)=\left(\begin{array}{ll}
\bar{\rho}_{e e} & \bar{\rho}_{\mu e} \\
\bar{\rho}_{e \mu} & \bar{\rho}_{\mu \mu}
\end{array}\right)=\frac{1}{2}\left[\bar{P}_{0}(p, t)+\boldsymbol{\sigma} \cdot \overline{\mathbf{P}}(p, t)\right] .
\end{aligned}
$$

Here, $\sigma_{i}$ are the Pauli matrices while $\mathbf{P}(p, t)$ and $\overline{\mathbf{P}}(p, t)$ are the usual polarization vectors for the neutrino and anti-neutrino modes $p$, respectively. We normalize $\rho$ and $\bar{\rho}$ to a Fermi-Dirac distribution with zero chemical potential, $f(p)=[\exp (p / T)+1]^{-1}$, so that for instance $f_{\nu_{a}}(p, t)=\rho_{a a}(p, t) f(p)$. For the polarization vectors, the equations of motion are given by the usual spin-precession formula

$$
\begin{aligned}
\partial_{t} \mathbf{P}_{p}=+\left[\frac{\Delta m^{2}}{2 p} \mathbf{B}-\frac{8 \sqrt{2} G_{\mathrm{F}} p}{3 m_{\mathrm{W}}^{2}} E_{e e} \hat{\mathbf{z}}\right] & \times \mathbf{P}_{p} \\
& +\sqrt{2} G_{\mathrm{F}}(\mathbf{P}-\overline{\mathbf{P}}) \times \mathbf{P}_{p}+C\left[\mathbf{P}_{p}\right], \\
\partial_{t} \overline{\mathbf{P}}_{p}=-\left[\frac{\Delta m^{2}}{2 p} \mathbf{B}-\frac{8 \sqrt{2} G_{\mathrm{F}} p}{3 m_{\mathrm{W}}^{2}} E_{e e} \hat{\mathbf{z}}\right] & \times \overline{\mathbf{P}}_{p} \\
& +\sqrt{2} G_{\mathrm{F}}(\mathbf{P}-\overline{\mathbf{P}}) \times \overline{\mathbf{P}}_{p}+C\left[\overline{\mathbf{P}}_{p}\right],
\end{aligned}
$$

where $\Delta m^{2}=m_{2}^{2}-m_{1}^{2}$ and $\mathbf{B}=(\sin 2 \theta, 0,-\cos 2 \theta)$ with $\theta$ the vacuum mixing angle. Further, $\hat{\mathbf{z}}$ is a unit vector in the $z$-direction, $E_{e e}$ the electron-positron 
energy density, and $\mathbf{P}$ and $\overline{\mathbf{P}}$ are the integrated polarization vectors.

We will see that a detailed treatment of the collision terms is not crucial. Therefore, we approximate them with a simple damping prescription of the form

$$
C\left[\mathbf{P}_{p}\right]=-D_{p} \mathbf{P}_{p}^{\mathrm{T}}
$$

where the transverse part $\mathbf{P}_{p}^{\mathrm{T}}$ consists of the $x$ - $y$-projection of $\mathbf{P}_{p}$. We use the damping functions as, for instance, in $[28,30]$, but with slightly modified coefficients. We neglect a small dependence on $\xi$, i.e. we use the same damping functions for neutrinos and antineutrinos. We have checked that our results are insensitive to the inclusion of the repopulation function, i.e. $\dot{P}_{0}$ and $\dot{P}_{0}$ as given for instance in $[28,30]$. Moreover, we did not include the neutrino heating from $e^{+} e^{-}$annihilations, since it is small even in the presence of degeneracies [31].

\subsection{Evolution of simple flavor asymmetry}

As a first case we consider a simple situation where initially electron neutrinos do not have a chemical potential $\left(\xi_{e}=0\right)$ while muon neutrinos are asymmetric $\left(\xi_{\mu}=-0.05\right)$. We use a rather small $\nu_{\mu}$ asymmetry so that the anticipated equilibrium state $\xi_{e}=\xi_{\mu}$ will be close to the BBN limit on $\xi_{e}$ in Eq. (2). The quantitative evolution with a large $\xi_{\mu}$ is the same. Moreover, we use maximal mixing and $\Delta m^{2}=4.5 \times 10^{-5} \mathrm{eV}^{2}$ as suggested by the LMA solution of the solar neutrino problem.

In order to illustrate the relative importance of the various contributions to the equation of motion we first calculate the primordial evolution of the integrated polarization vectors $\mathbf{P}$ and $\overline{\mathbf{P}}$ for pure vacuum oscillations, including only the $\Delta m^{2} / 2 p$ terms in Eq. (7), without any medium effects whatsoever. Our initial conditions $\xi_{e}=0$ and $\xi_{\mu}=-0.05$ imply that there are more $\nu_{e}$ than $\nu_{\mu}$, i.e. $\mathbf{P}$ points initially in the $+\hat{\mathbf{z}}$ direction, while there are more $\bar{\nu}_{\mu}$ than $\bar{\nu}_{e}$, i.e. $\overline{\mathbf{P}}$ points initially in the $-\hat{\mathbf{z}}$ direction. In the upper panel of Fig. 1 we show the evolution of $P_{z}$ as a function of cosmic temperature; the evolution of $\bar{P}_{z}$ is similar, except with a negative initial value. The oscillations begin when the expansion rate has become slow enough at about $T \simeq 30 \mathrm{MeV}$. The oscillation frequencies are different for different modes, leading to quick decoherence and thus to an incoherent equal mix of both flavors. Evidently flavor equilibrium is reached long before $n / p$ freeze-out at $T \simeq 1 \mathrm{MeV}$.

As a next step we include the neutrino self-potential proportional to $\mathbf{P}-\overline{\mathbf{P}}$. The evolution is shown as a dotted line in the upper panel of Fig. 1. It is now dominated by the effect of synchronized oscillations, i.e. the self-potential 
forces all neutrino modes to follow the same oscillation [26]. Because $\mathbf{P}$ and $\overline{\mathbf{P}}$ point in opposite directions, the common or synchronized oscillation frequency $\omega_{\text {synch }}$ is very much smaller than a typical $\Delta m^{2} / 2 p$. Explicitly we have [26]

$$
\omega_{\text {synch }}=\frac{1}{|\mathbf{I}|} \int \frac{d^{3} p}{(2 \pi)^{3}} f(p) \frac{\Delta m^{2}}{2 p} \hat{\mathbf{I}} \cdot\left(\mathbf{P}_{p}+\overline{\mathbf{P}}_{p}\right) \equiv \frac{\Delta m^{2}}{2 p_{\mathrm{eff}}}
$$

where $\mathbf{I} \equiv \mathbf{P}-\overline{\mathbf{P}}$ and $\hat{\mathbf{I}}$ is a unit vector in the direction of $\mathbf{I}$. Numerically our initial conditions imply $p_{\text {eff }} \simeq 132 T$.

Next we add to the vacuum term the effective potential caused by the $e^{ \pm}$background [see Eq. (4)], without including the self-term. The $e^{ \pm}$potential always points in the $z$-direction, thereby suppressing flavor oscillations in the usual way. This effect is shown in the lower panel of Fig. 1 (solid line). $\mathbf{P}$ stays frozen at its initial value up to $T \simeq 3 \mathrm{MeV}$ where the medium potential becomes unimportant compared to the vacuum term. At these temperatures collisions are also about to become unimportant. Therefore it is not surprising that including collisions (dotted line) does not dramatically change the evolution of $P_{z}$.

However, this impression is misleading as can be seen from Fig. 2 where we show the evolution of $P_{x}$ for the same cases as in the lower panel of Fig. 1. Without collisions (solid line) the evolution of $\mathbf{P}$ is a simple turning from the $z$ - to the $x$-direction. The $e^{ \pm}$potential adiabatically disappears, leading to the usual MSW-type evolution. In this case the final result corresponds to equal numbers of both flavors, yet a coherent flavor superposition. With collisional damping (dotted line) the $x$-component is damped, leaving little flavor coherence of the final state. Therefore, with or without collisional damping we reach "flavor equilibrium" in the sense of equal densities of both flavors, but only collisions ensure the damping of the otherwise large transverse part of the final $\mathbf{P}$.

Finally we study how oscillations evolve in the presence of all effects: vacuum, $e^{ \pm}$background, neutrino self-potential and collisions. The results are shown in Fig. 3 for different strengths of the self-potential. In one run it was switched off ("No Self"), it was taken at full strength ("Self"), or it was suppressed by a factor $10^{4}$ or $10^{5}$. When the self-interaction term is close to its real value (solid line), again all modes oscillate in synch so that we see a combination of the synchronized oscillations and the MSW-like effect of the background medium. However, collisions ensure that no large transverse component survives - see the corresponding evolution of $P_{x}$ and $P_{y}$ in Fig. 4. Almost perfect flavor equilibrium is achieved with or without self-interactions.

This conclusion is not to be taken for granted. When the self-interaction term is artificially adjusted to be of comparable strength to the other terms (vacuum 
or $e^{ \pm}$background), the evolution shows complicated features (Fig. 3). After some initial conversion, $P_{z}$ remains constant for a long time, only to reach equilibrium at a much later time. This behavior is not easy to understand as it depends on all the ingredients of our calculation, i.e. the background potential, the neutrino self-potential and damping by collisions. We believe that the long phase where the polarization vector essentially stands still is caused by the synchronized oscillation frequency becoming very small. This is strictly the case in a situation where the chemical potentials for two flavors are equal but opposite - see Sec. 3.3 below. We believe that in the present case a combination of decoherence of some of the modes and collisions drives the system into a state where the synchronized oscillations frequency vanishes so that oscillations stop until the cosmic expansion has diluted the neutrino self-term enough to eliminate this effect.

\subsection{Equal but opposite asymmetries}

Our discussion thus far suggests that for the parameters of interest it is generic to achieve flavor equilibrium before the BBN epoch, even though the loss of coherence may not always be complete. However, this conclusion depends on the simple initial conditions that we have used thus far. The different neutrino flavors may show large asymmetries, yet the total cosmic lepton asymmetry could still be small if initially $\xi_{e}=-\xi_{\mu}$, which corresponds to conservation of the lepton number $L_{e}-L_{\mu}$. These initial conditions imply that $\mathbf{P}=-\overline{\mathbf{P}}$ so that the self-potential term $(\mathbf{P}-\overline{\mathbf{P}})$ is large while for each mode $\left(\mathbf{P}_{p}+\overline{\mathbf{P}}_{p}\right)=0[26]$. This implies, in turn, that the synchronized oscillation frequency $\omega_{\text {synch }}=0$. Therefore, in this case the synchronization effect caused by the self-potential prevents flavor oscillations entirely, at least until the self-term becomes weak, long after the BBN epoch. While flavor equilibrium is not achieved in this

case, from the start we have $\left|\xi_{e}\right|=\left|\xi_{\mu}\right|$ so that the BBN limits on $\left|\xi_{e}\right|$ would apply to both flavors.

\section{Three-flavor oscillations}

\subsection{Oscillation parameters}

We now turn to the more interesting case of three-flavor neutrino oscillations. The neutrino flavor eigenstates $\nu_{e}, \nu_{\mu}$, and $\nu_{\tau}$ are related to the mass eigen- 
states via the mixing matrix

$$
\left(\begin{array}{ccc}
c_{12} c_{13} & s_{12} c_{13} & s_{13} \\
-s_{12} c_{23}-c_{12} s_{23} s_{13} & c_{12} c_{23}-s_{12} s_{23} s_{13} & s_{23} c_{13} \\
s_{12} s_{23}-c_{12} c_{23} s_{13} & -c_{12} s_{23}-s_{12} c_{23} s_{13} & c_{23} c_{13}
\end{array}\right) .
$$

Here $c_{i j}=\cos \theta_{i j}$ and $s_{i j}=\sin \theta_{i j}$ for $i j=12,23$, or 13 , and we have assumed $\mathrm{CP}$ conservation. The set of oscillation parameters is now five-dimensional (see for instance [32]),

$$
\begin{aligned}
& \Delta m_{\mathrm{sun}}^{2} \equiv \Delta m_{21}^{2}=m_{2}^{2}-m_{1}^{2} \\
& \Delta m_{\mathrm{atm}}^{2} \equiv \Delta m_{32}^{2}=m_{3}^{2}-m_{2}^{2} \\
& \theta_{\text {sun }} \equiv \theta_{12} \\
& \theta_{\text {atm }} \equiv \theta_{23} \\
& \theta_{13}
\end{aligned}
$$

We do not perform a global analysis of all possible values of these parameters, but fix them to be in the regions that solve the atmospheric and solar neutrino problems $[32,33]$. In particular we take $\Delta m_{\text {atm }}^{2}=3 \times 10^{-3} \mathrm{eV}^{2}$ and maximal mixing for $\theta_{\text {atm }}$ from the former, while from the solar analyses we consider the following values for $\Delta m_{\text {sun }}^{2}$ in $\mathrm{eV}^{2}: 4.5 \times 10^{-5}, 7 \times 10^{-6}, 1 \times 10^{-7}, 8 \times 10^{-11}$ for the Large Mixing Angle (LMA), Small Mixing Angle (SMA), LOW and Vacuum regions, respectively. For the angle $\theta_{\text {sun }}$ we take the approximation of maximal mixing for all cases except SMA where we use $\theta_{\text {sun }}=1.5^{\circ}$.

\subsection{Simple three flavor case}

We begin with the simplified situation where initially only the muon neutrinos are asymmetric $\left(\xi_{\mu}=-0.1\right)$. We perform a full three-flavor calculation, but for now set $\theta_{13}=0$. The evolution of the neutrino asymmetries is shown in Figs. 5 and 6 for the LMA and LOW cases, respectively, both with and without the neutrino self-interactions. For this choice of oscillation parameters the three-flavor oscillations effectively separate as two two-flavor problems for the atmospheric and solar parameters, respectively. The oscillations caused by the largest $\Delta m^{2}$ are effective at $T \simeq 20 \mathrm{MeV}$, as soon as the $\mu^{ \pm}$background disappears completely. The presence of the self-term causes only a slight delay in the equilibration of $\xi_{\mu}$ and $\xi_{\tau}$.

The oscillations due to $\Delta m_{\text {sun }}^{2}$ and $\theta_{\text {sun }}$ are effective only when the vacuum term 
overcomes the $e^{ \pm}$potential. In the LMA case, the conversions takes place above $T \simeq 1 \mathrm{MeV}$, leading to nearly complete flavor equilibrium before the onset of BBN. For the LOW parameters the synchronized oscillations just start at that epoch. The presence of the neutrino self-potential does not significantly change the picture in the LMA case while for the LOW case one clearly observes the phenomenon of synchronized oscillations. For the SMA and Vacuum regions primordial oscillations involving $\nu_{e}$ are not effective before BBN if $\theta_{13}=0$.

\section{$4.3 \quad$ Non-zero 13 mixing}

The angle $\theta_{13}$ is restricted to the approximate region $\tan ^{2} \theta_{13} \lesssim 0.065$ (see for instance [34]) from a combined analysis of solar, atmospheric and reactor (CHOOZ) data. However, a small but non-zero $\theta_{13}$ does modify the oscillation behavior. This effect is shown in Fig. 7 for the LMA case and different values of $\theta_{13}$. Even small values of $\theta_{13}$ lead to conversion to the electronic flavor at larger temperatures and enhance flavor equilibration if $\theta_{\text {sun }}$ is in the LMA region.

The effect of a non-zero $\theta_{13}$ is more important if the solar neutrino problem is solved by oscillations with parameters in a region other than LMA. This can be seen in Fig. 8, where we have calculated the evolution in the limit $\Delta m_{\text {sun }}^{2} \simeq 0$ and $\theta_{\text {sun }} \simeq 0$, thus corresponding approximately to the SMA, LOW and Vacuum regions. For values of $\tan ^{2} \theta_{13}$ close to the limit discussed at the beginning of this section, neutrino oscillations lead to almost complete flavor equilibration, while for smaller $\theta_{13}$ angles the conversion is only partial.

\subsection{Can the self-term prevent flavor equilibrium?}

In the two-flavor case of Sec. 3.3 we saw that for equal but opposite asymmetries of the two flavor distributions the synchronized oscillation frequency was strictly zero, suppressing oscillations entirely before the BBN epoch. In the three flavor case the situation is more complicated so that again we need to raise the question if there is a special configuration of initial neutrino distributions which suppresses flavor oscillations by the neutrino self-potential.

In analogy to Sec. 3.3 we first consider situations where the asymmetries of two flavors are equal but opposite, while the third asymmetry is strictly zero. In this case one expects that oscillations between the asymmetric flavors are suppressed by the self-potential while oscillations into the third flavor are unimpeded. We show the numerical results for two specific cases. In Fig. 9 we take $\xi_{\mu}=-\xi_{\tau}=-0.1$ and $\xi_{e}=0$. The "atmospheric" oscillations between $\nu_{\mu}$ and $\nu_{\tau}$ are indeed blocked in the presence of self-interactions while the "solar 
oscillations", here the LMA case and $\tan ^{2} \theta_{13}=0$, proceed at the appropriate temperature. In Fig. 10 we take $\xi_{\tau}=0$ and $\xi_{e}=-\xi_{\mu}=0.1$. Both the atmospheric and solar oscillations take place and equilibrate the flavors essentially as in the previous cases with a simple initial condition.

The question remains if one could devise special initial conditions such that $\left|\xi_{\tau}\right|$ and $\left|\xi_{\mu}\right|$ are large while $\left|\xi_{e}\right|$ is small, and that this system avoids flavor oscillations by reducing the synchronized oscillation frequency to a very small value. In this case our new limits would not apply.

We do not believe that such a case can be constructed. To argue in favor of this claim we first note that the most general initial condition consists of thermal equilibrium distributions which are diagonal in flavor space, i.e. in the weak-interaction basis the initial $3 \times 3$ density matrices for neutrinos and anti-neutrinos are diagonal and characterized by Fermi-Dirac distributions. All off-diagonal elements would be quickly damped by reactions which involve muons and electrons and thus "measure" the flavor content of the participating neutrinos. Therefore, the most natural initial condition for our problem is indeed characterized by $\xi_{e}, \xi_{\mu}$ and $\xi_{\tau}$.

The general equations of motion Eq. (4) reveal that the quantity which develops in a synchronized fashion is $\rho-\bar{\rho}$, in agreement with the discussion of $2 \times 2$ synchronized oscillations in Ref. [26] where the picture of polarization vectors and the associated "internal magnetic fields" of the system was used. Therefore, the synchronized equation of motion is obtained by subtracting the two lines of Eq. (4) and integrating over all modes,

$$
\begin{aligned}
i \partial_{t}(\rho-\bar{\rho})= & \int \frac{d^{3} p}{(2 \pi)^{3}}\left[\frac{M^{2}}{2 p},\left(\rho_{p}+\bar{\rho}_{p}\right)\right] \\
& -\sqrt{2} G_{\mathrm{F}} \int \frac{d^{3} p}{(2 \pi)^{3}}\left[\frac{8 p}{3 m_{\mathrm{W}}^{2}} E,\left(\rho_{p}+\bar{\rho}_{p}\right)\right] \\
& +\sqrt{2} G_{\mathrm{F}}[(\rho-\bar{\rho}),(\rho-\bar{\rho})],
\end{aligned}
$$

where we have dropped the collision term. The last term, of course, is identically zero. The second term vanishes also as long as the density matrices are diagonal in the flavor basis since the matrix $E$ is diagonal in that basis. Only the first term, involving the mass matrix, represents a "force" such as to move $\rho-\bar{\rho}$ away from being diagonal in the flavor basis, i.e. which causes synchronized flavor oscillations. This force is identically zero if the matrix

$$
\int \frac{d^{3} p}{(2 \pi)^{3}} \frac{\rho_{p}+\bar{\rho}_{p}}{2 p}
$$

is proportional to the unit matrix. As the matrices $\rho_{p}$ and $\bar{\rho}_{p}$ are initially 
diagonal and given by Fermi-Dirac distributions, the integral can be solved explicitly, leading to an expression proportional to

$$
\left(\begin{array}{lll}
1 & & \\
& 1 & \\
& & 1
\end{array}\right)+\frac{3}{\pi^{2}}\left(\begin{array}{ccc}
\xi_{e}^{2} & & \\
& \xi_{\mu}^{2} & \\
& & \xi_{\tau}^{2}
\end{array}\right) .
$$

If all $\xi$ are initially equal, then the neutrinos are already in flavor equilibrium and indeed oscillations trivially do not operate.

The only nontrivial configuration where synchronized oscillations proceed with a vanishing frequency is when one of the chemical potentials is opposite in sign to the others. We have checked numerically that indeed no flavor conversion arises in this case as long as the self-potential is large, i.e. as long as we are in the synchronized regime. In the two-flavor case this corresponds to the situation discussed in Sec. 3.3.

As in the two-flavor case we conclude that it is possible to avoid flavor equilibrium by specially chosen initial conditions, but these conditions require $\left|\xi_{e}\right|=\left|\xi_{\mu}\right|=\left|\xi_{\tau}\right|$. Therefore, the strict BBN limits on $\left|\xi_{e}\right|$ apply to all flavors.

\section{New limits on neutrino degeneracy}

We conclude that in the LMA region the neutrino flavors essentially equilibrate long before $n / p$ freeze out, even when $\theta_{13}$ is vanishingly small. For the other cases the outcome depends on the magnitude of $\theta_{13}$. In the LMA case it is thus justified to derive new limits on the cosmic neutrino degeneracy parameters under the assumption that all three neutrino flavors are characterized by a single degeneracy parameter, independently of the primordial initial conditions. We do not derive the corresponding limits for the other solar neutrino solutions, since they would strongly depend on the value of a non-zero $\theta_{13}$. However, if that angle is close to the experimental limit, the bounds that we describe would approximately apply.

We first note that the energy density in one species of neutrinos and antineutrinos with degeneracy parameter $\xi$ is

$$
\rho_{\nu \bar{\nu}}=T_{\nu}^{4} \frac{7 \pi^{2}}{120}\left[1+\frac{30}{7}\left(\frac{\xi}{\pi}\right)^{2}+\frac{15}{7}\left(\frac{\xi}{\pi}\right)^{4}\right] .
$$

It is clear that the BBN limit will imply $\xi \ll 1$ for all flavors so that the modified energy density and the resulting change of the primordial helium abun- 
dance $Y_{\mathrm{p}}$ will be negligibly small. If there are additional relativistic species, such as sterile neutrinos or majorons, then Eq. (2) will simply apply to all the active neutrinos

$$
|\xi|<0.22
$$

Therefore, the only remaining BBN effect is the shift of the beta equilibrium by $\xi_{e}$. We recall that $Y_{\mathrm{p}}$ is essentially given by $n / p$ at the weak-interaction freezeout, and that $n / p \propto \exp \left(-\xi_{e}\right) \simeq 1-\xi_{e}$ where the latter expansion applies for $\left|\xi_{e}\right| \ll 1$. Therefore, $\Delta Y_{\mathrm{p}} \simeq-Y_{\mathrm{p}}\left(1-Y_{\mathrm{p}} / 2\right) \xi_{e} \simeq-0.21 \xi_{e}$. Modifications of $Y_{\mathrm{p}}$ by new physics are frequently expressed in terms of the equivalent number of neutrino flavors $\Delta N_{\nu}$ which would cause the same modification due to the changed expansion rate at $\mathrm{BBN}$. If the radiation density at $\mathrm{BBN}$ is expressed in terms of $N_{\nu}$, the helium yield can be expressed by the empirical formula $\Delta Y_{\mathrm{p}}=$ $0.012 \Delta N_{\nu}$ [35]. Therefore, the effect of a small $\xi_{e}$ on the helium abundance is equivalent to $\Delta N_{\nu} \simeq-18 \xi_{e}$. A conservative standard limits holds that BBN implies $\left|\Delta N_{\nu}\right|<1$ which thus translates into $\left|\xi_{e}\right| \lesssim 0.057$.

A more detailed recent analysis reveals that the measured primordial helium abundance implies a $95 \%$ CL range $N_{\nu}=2.5 \pm 0.8$ or $\Delta N_{\nu}=-0.5 \pm 0.8[8,13]$. We conclude that the BBN-favored range for the electron neutrino degeneracy parameter is at $95 \% \mathrm{CL}$

$$
\xi_{e}=0.03 \pm 0.04
$$

If all degeneracy parameters are the same, then this range applies to all flavors.

It should be noted that the actual limit we obtain on the neutrino degeneracy depends on the adopted BBN analysis. For instance $\Delta N_{\nu}$ could be as high as 1.2 when the primordial abundance of lithium is used instead of that of deuterium [36]. At any rate, a limit of $\left|\xi_{e}\right| \lesssim 0.1$ seems rather conservative and does not modify our conclusions.

Using $|\xi|<0.07$ as a limit on the one degeneracy parameter for all flavors, the extra radiation density is limited by $\left(\Delta \rho_{\nu \bar{\nu}}\right) / \rho_{\nu \bar{\nu}}<3 \times 0.0021=0.0064$, i.e. $\Delta N_{\nu}<0.0064$. If the same radiation density were to be produced by the asymmetry of one single species, this would correspond to $|\xi|<0.12$.

For comparison with the future satellite experiments MAP and PLANCK that will measure the CMBR anisotropies, it was calculated that they optimistically will be sensitive to a single-species $\xi$ above 0.5 and 0.25 , respectively [37]. However, with proper consideration of the degeneracy with the matter density, $\omega_{M}$, and the spectral index, $n$, a more realistic sensitivity is $\xi \approx 2.4$ and 0.73 , respectively [38]. Turning this around we conclude that our new limits are so restrictive that the CMBR is certain to remain unaffected by neutrino 
degeneracy effects so that $|\xi|$ can be safely neglected as a fit parameter in future analyses.

If our new limits apply the number density of relic neutrinos is very close to its standard value. Therefore, existing limits and possible future measurements of the absolute neutrino mass scale, for example in the forthcoming tritium decay experiment KATRIN [39], will provide unambiguous information on the cosmic mass density in neutrinos, free of the uncertainty of neutrino chemical potentials.

Note added: Very recently the authors of refs. [40,41] have also analyzed the equilibration of neutrino asymmetry from flavor oscillations, providing further analytical insight and confirming our conclusions.

\section{Acknowledgments}

We thank Alexei Smirnov, Gary Steigman, Karsten Jedamzik and Gianpiero Mangano for useful discussions and comments. In Munich, this work was partly supported by the Deutsche Forschungsgemeinschaft under grant No. SFB 375 and the ESF network Neutrino Astrophysics. S.H. Hansen and S. Pastor are supported by Marie Curie fellowships of the European Commission under contracts HPMFCT-2000-00607 and HPMFCT-2000-00445.

\section{A Evolution equations}

In this appendix we list in detail the evolution equations for the neutrino and anti-neutrino density matrices in the two-flavor case as in Eq. (5), while the generalization to the three-flavor case that we consider in Sec. 4 is straightforward.

In our treatment of primordial neutrino oscillations we use the following dimensionless expansion rate and momenta

$$
x \equiv m R, \quad y \equiv p R
$$

where $R$ is the universe scale factor and $m$ an arbitrary mass scale that we choose to be $1 \mathrm{MeV}$. The neutrino and anti-neutrino density matrices are

$$
\rho(x, y)=\frac{1}{2}\left[P_{0}(x, y)+\boldsymbol{\sigma} \cdot \mathbf{P}(x, y)\right]=\frac{1}{2}\left(\begin{array}{cc}
P_{0}+P_{z} & P_{x}-i P_{y} \\
P_{x}+i P_{y} & P_{0}-P_{z}
\end{array}\right)
$$




$$
\bar{\rho}(x, y)=\frac{1}{2}\left[\bar{P}_{0}(x, y)+\boldsymbol{\sigma} \cdot \overline{\mathbf{P}}(x, y)\right]=\frac{1}{2}\left(\begin{array}{ll}
\bar{P}_{0}+\bar{P}_{z} & \bar{P}_{x}-i \bar{P}_{y} \\
\bar{P}_{x}+i \bar{P}_{y} & \bar{P}_{0}-\bar{P}_{z}
\end{array}\right)
$$

with the derivatives

$$
H x \frac{d \rho}{d x}(x, y)=\frac{d \rho}{d t}(t, p), \quad H x \frac{d \bar{\rho}}{d x}(x, y)=\frac{d \bar{\rho}}{d t}(t, p) .
$$

The initial conditions are for large temperatures as follows. For density matrices normalized to $f_{\text {eq }}(y)=\left(e^{y}+1\right)^{-1}$ and initial degeneracies $\xi_{\alpha}=-\xi_{\bar{\alpha}}$ and $\xi_{\beta}=-\xi_{\bar{\beta}}$ (for flavor neutrinos $\nu_{\alpha}$ and $\nu_{\beta}$ )

$$
\begin{aligned}
P_{0}(y) & =\frac{f_{\mathrm{eq}}\left(y, \xi_{\alpha}\right)+f_{\mathrm{eq}}\left(y, \xi_{\beta}\right)}{f_{\mathrm{eq}}(y)} \\
P_{z}(y) & =\frac{f_{\mathrm{eq}}\left(y, \xi_{\alpha}\right)-f_{\mathrm{eq}}\left(y, \xi_{\beta}\right)}{f_{\mathrm{eq}}(y)} \\
\bar{P}_{0}(y) & =\frac{f_{\mathrm{eq}}\left(y,-\xi_{\alpha}\right)+f_{\mathrm{eq}}\left(y,-\xi_{\beta}\right)}{f_{\mathrm{eq}}(y)} \\
\bar{P}_{z}(y) & =\frac{f_{\mathrm{eq}}\left(y,-\xi_{\alpha}\right)-f_{\mathrm{eq}}\left(y,-\xi_{\beta}\right)}{f_{\mathrm{eq}}(y)}
\end{aligned}
$$

where $f_{\text {eq }}(y, \xi)=1 /[\exp (y-\xi)+1]$. Finally,

$$
P_{x}(y)=P_{y}(y)=\bar{P}_{x}(y)=\bar{P}_{y}(y)=0 .
$$

The components of the neutrino polarization vectors evolve as

$$
\begin{aligned}
\frac{d P_{0}}{d x}(x, y)= & R_{\alpha}(x, y)+R_{\beta}(x, y) \\
\frac{d P_{x}}{d x}(x, y)= & \left(\frac{\Delta m^{2}}{2 p H x} \cos 2 \theta-\frac{V_{l}}{H x}\right) P_{y}(x, y)-\frac{D}{H x} P_{x}(x, y) \\
\frac{d P_{y}}{d x}(x, y)= & -\left(\frac{\Delta m^{2}}{2 p H x} \cos 2 \theta-\frac{V_{l}}{H x}\right) P_{x}(x, y)-\frac{D}{H x} P_{y}(x, y) \\
& -\frac{\Delta m^{2}}{2 p H x} \sin 2 \theta P_{z}(x, y) \\
\frac{d P_{z}}{d x}(x, y)= & \frac{\Delta m^{2}}{2 p H x} \sin 2 \theta P_{y}(x, y)+R_{\alpha}(x, y)-R_{\beta}(x, y) \\
\frac{d \bar{P}_{0}}{d x}(x, y)= & \bar{R}_{\alpha}(x, y)+\bar{R}_{\beta}(x, y) \\
\frac{d \bar{P}_{x}}{d x}(x, y)= & -\left(\frac{\Delta m^{2}}{2 p H x} \cos 2 \theta-\frac{V_{l}}{H x}\right) \bar{P}_{y}(x, y)-\frac{D}{H x} \bar{P}_{x}(x, y)
\end{aligned}
$$




$$
\begin{aligned}
\frac{d \bar{P}_{y}}{d x}(x, y)= & \left(\frac{\Delta m^{2}}{2 p H x} \cos 2 \theta-\frac{V_{l}}{H x}\right) \bar{P}_{x}(x, y)-\frac{D}{H x} \bar{P}_{y}(x, y) \\
& +\frac{\Delta m^{2}}{2 p H x} \sin 2 \theta \bar{P}_{z}(x, y) \\
\frac{d \bar{P}_{z}}{d x}(x, y)= & -\frac{\Delta m^{2}}{2 p H x} \sin 2 \theta \bar{P}_{y}(x, y)+\bar{R}_{\alpha}(x, y)-\bar{R}_{\beta}(x, y)
\end{aligned}
$$

where

$$
\begin{aligned}
& R_{\alpha}(x, y)=2 \frac{D}{H x}\left[\frac{f_{\mathrm{eq}}\left(y, \xi_{\alpha}\right)}{f_{\mathrm{eq}}(y)}-\frac{1}{2}\left(P_{0}(x, y)+P_{z}(x, y)\right)\right] \\
& R_{\beta}(x, y)=2 \frac{D}{H x}\left[\frac{f_{\mathrm{eq}}\left(y, \xi_{\beta}\right)}{f_{\mathrm{eq}}(y)}-\frac{1}{2}\left(P_{0}(x, y)-P_{z}(x, y)\right)\right],
\end{aligned}
$$

with $\bar{R}_{\alpha, \beta}$ given by the same expressions with $\xi \rightarrow-\xi$. However, our numerical results do not change significantly if the total neutrino and anti-neutrino number densities are taken to be constant $\left(d P_{0} / d x=d \bar{P}_{0} / d x=0\right)$.

The different terms in Eqs. (A.7)are given as follows. The vacuum oscillation terms are proportional to

$$
\frac{\Delta m^{2}}{2 p H x}=\frac{10^{10} M_{P}}{2 \sqrt{8 \pi / 3}}\left(\frac{\Delta m^{2}}{\mathrm{eV}^{2}}\right) \frac{1}{\sqrt{\rho}} \frac{x^{2}}{y}
$$

where $M_{P} \equiv 1.221, \bar{\rho}=(x / m)^{4} \rho_{\text {tot }}$, and $\rho_{\text {tot }}$ is the total energy density of the universe. The $l^{+} l^{-}$background with $l=\mu$,e the charged leptons (for $\nu_{\tau^{-}} \nu_{\mu}$ and $\nu_{e}-\nu_{\mu, \tau}$ oscillations, respectively) is described by

$$
\frac{V_{l}}{H x}=-\frac{8 \sqrt{2} 10^{5} M_{P} G_{F}}{3 \sqrt{8 \pi / 3} m_{W}^{2}} \frac{1}{\sqrt{\bar{\rho}}} \frac{y}{x^{4}}\left(\bar{\rho}_{l^{+}}+\bar{\rho}_{l^{-}}\right)
$$

where $G_{F} \equiv 1.1664$ and $m_{W} \equiv 80.42$. Collisions are described by damping terms where, according to our calculations,

$$
\begin{aligned}
& D \simeq 2 \times\left(4 \sin ^{4} \theta_{W}-2 \sin ^{2} \theta_{W}+2\right) F_{0}, \\
& D \simeq 2 \times\left(2 \sin ^{4} \theta_{W}+6\right) F_{0},
\end{aligned}
$$

for $\nu_{\tau}-\nu_{\mu}$ and $\nu_{e}-\nu_{\mu, \tau}$ oscillations, respectively, where all collision terms are proportional to

$$
\frac{F_{0}}{H x}=\frac{M_{P} G_{F}^{2} \zeta(3) y_{\mathrm{med}}}{3 \pi^{3} \sqrt{8 \pi / 3}} \frac{1}{\sqrt{\bar{\rho}}} \frac{y}{x^{4}}
$$


and $\zeta(3) \simeq 1.20206$ and $y_{\text {med }} \simeq 3.15137$

Finally one has to add the contribution from the neutrino-antineutrino background [27-29]

$$
\begin{aligned}
& {\left[\frac{V_{\text {asym }}}{H x}(\mathbf{J}(x)-\overline{\mathbf{J}}(x))-\frac{V_{\text {sym }}}{H x}(\mathbf{U}(x)+\overline{\mathbf{U}}(x))\right] \times \mathbf{P}(x, y),} \\
& {\left[\frac{V_{\text {asym }}}{H x}(\mathbf{J}(x)-\overline{\mathbf{J}}(x))+\frac{V_{\text {sym }}}{H x}(\mathbf{U}(x)+\overline{\mathbf{U}}(x))\right] \times \overline{\mathbf{P}}(x, y),}
\end{aligned}
$$

for $d \mathbf{P} / d x$ and $d \overline{\mathbf{P}} / d x$, respectively. Here we have defined

$$
\mathbf{J}(x)=\frac{1}{2 \pi^{2}} \int d u u^{2} \mathbf{P}(x, u), \quad \mathbf{U}(x)=\frac{1}{2 \pi^{2}} \int d u u^{3} \mathbf{P}(x, u),
$$

and the same for $\overline{\mathbf{J}}$ and $\overline{\mathbf{U}}$. Moreover,

$$
\begin{aligned}
\frac{V_{\mathrm{asym}}}{H x} & =\frac{\sqrt{2} 10^{11} M_{P} G_{F}}{\sqrt{8 \pi / 3}} \frac{1}{\sqrt{\bar{\rho}} x^{2}} \\
\frac{V_{\mathrm{sym}}}{H x} & =\frac{8 \sqrt{2} 10^{5} M_{P} G_{F}}{3 \sqrt{8 \pi / 3} m_{W}^{2}} \cos ^{2} \theta_{W} \frac{1}{\sqrt{\bar{\rho}}} \frac{y}{x^{4}} .
\end{aligned}
$$

\section{References}

[1] W. Buchmüller and M. Plümacher, Int. J. Mod. Phys. A 15 (2000) 5047.

[2] J.A. Harvey and E.W. Kolb, Phys. Rev. D 24 (1981) 2090.

[3] A.D. Dolgov and D.P. Kirilova, J. Moscow Phys. Soc. 1 (1991) 217.

[4] A. Casas, W.Y. Cheng and G. Gelmini, Nucl. Phys. B 538 (1999) 297.

[5] A.D. Dolgov, Phys. Rept. 222 (1992) 309.

[6] S. Sarkar, Rept. Prog. Phys. 59 (1996) 1493.

[7] H. Kang and G. Steigman, Nucl. Phys. B 372 (1992) 494.

[8] S. Esposito, G. Mangano, G. Miele and O. Pisanti, JHEP 0009 (2000) 038.

[9] S. Esposito, G. Mangano, A. Melchiorri, G. Miele and O. Pisanti, Phys. Rev. D 63 (2001) 043004.

[10] P. Di Bari, Phys. Rev. D 65 (2002) 043509.

[11] J. Lesgourgues and S. Pastor, Phys. Rev. D 60 (1999) 103521. 
[12] W.Hu, D.J. Eisenstein, M. Tegmark and M.J. White, Phys. Rev. D 59 (1999) 023512 .

[13] S.H. Hansen, G. Mangano, A. Melchiorri, G. Miele and O. Pisanti, Phys. Rev. D 65 (2002) 023511.

[14] S. Hannestad, Phys. Rev. D 64 (2001) 083002.

[15] J.P. Kneller, R.J. Scherrer, G. Steigman and T.P. Walker, Phys. Rev. D 64 (2001) 123506.

[16] J. Lesgourgues and M. Peloso, Phys. Rev. D 62 (2000) 081301.

[17] G. Gelmini and A. Kusenko, Phys. Rev. Lett. 82 (1999) 5202.

[18] P.B. Pal and K. Kar, Phys. Lett. B 451 (1999) 136.

[19] J. Lesgourgues, S. Pastor and S. Prunet, Phys. Rev. D 62 (2000) 023001.

[20] C. Lunardini and A.Yu. Smirnov, Phys. Rev. D 64 (2001) 073006.

[21] A.D. Dolgov, S.H. Hansen and D.V. Semikoz, Nucl. Phys. B 503 (1997) 426.

[22] P. Langacker, S.T. Petcov, G. Steigman and S. Toshev, Nucl. Phys. B 282 (1987) 589.

[23] S. Hannestad, to be published in Phys. Rev. D astro-ph/0111423.

[24] M.J. Savage, R.A. Malaney and G.M. Fuller, Astrophys. J. 368 (1991) 1.

[25] S. Samuel, Phys. Rev. D 48 (1993) 1462.

[26] S. Pastor, G.G. Raffelt and D.V. Semikoz, Phys. Rev. D 65 (2002) 053011.

[27] G. Sigl and G. Raffelt, Nucl. Phys. B 406 (1993) 423.

[28] B.H. McKellar and M.J. Thomson, Phys. Rev. D 49 (1994) 2710.

[29] J. Pantaleone, Phys. Lett. B 287 (1992) 128.

[30] N.F. Bell, R.R. Volkas and Y.Y. Wong, Phys. Rev. D 59 (1999) 113001.

[31] S. Esposito, G. Miele, S. Pastor, M. Peloso and O. Pisanti, Nucl. Phys. B 590 (2000) 539 .

[32] M.C. González-García, M. Maltoni, C. Peña-Garay and J.W.F. Valle, Phys. Rev. D 63 (2001) 033005.

[33] G.L. Fogli, E. Lisi, A. Marrone, D. Montanino and A. Palazzo, hep$\mathrm{ph} / 0104221$.

[34] A. Bandyopadhyay, S. Choubey, S. Goswami and K. Kar, hep-ph/0110307.

[35] R.A. Malaney and G.J. Mathews, Phys. Rept. 229 (1993) 145.

[36] K.A. Olive, astro-ph/0202486. 
[37] W.H. Kinney and A. Riotto, Phys. Rev. Lett. 83 (1999) 3366.

[38] R. Bowen, S.H. Hansen, A. Melchiorri, J. Silk and R. Trotta, to be published in $M N R A S$ astro-ph/0110636].

[39] A. Osipowicz et al. [KATRIN Collaboration], hep-ex/0109033.

[40] Y.Y.Y. Wong, hep-ph/0203180.

[41] K.N. Abazajian, J.F. Beacom and N.F. Bell, astro-ph/0203442. 


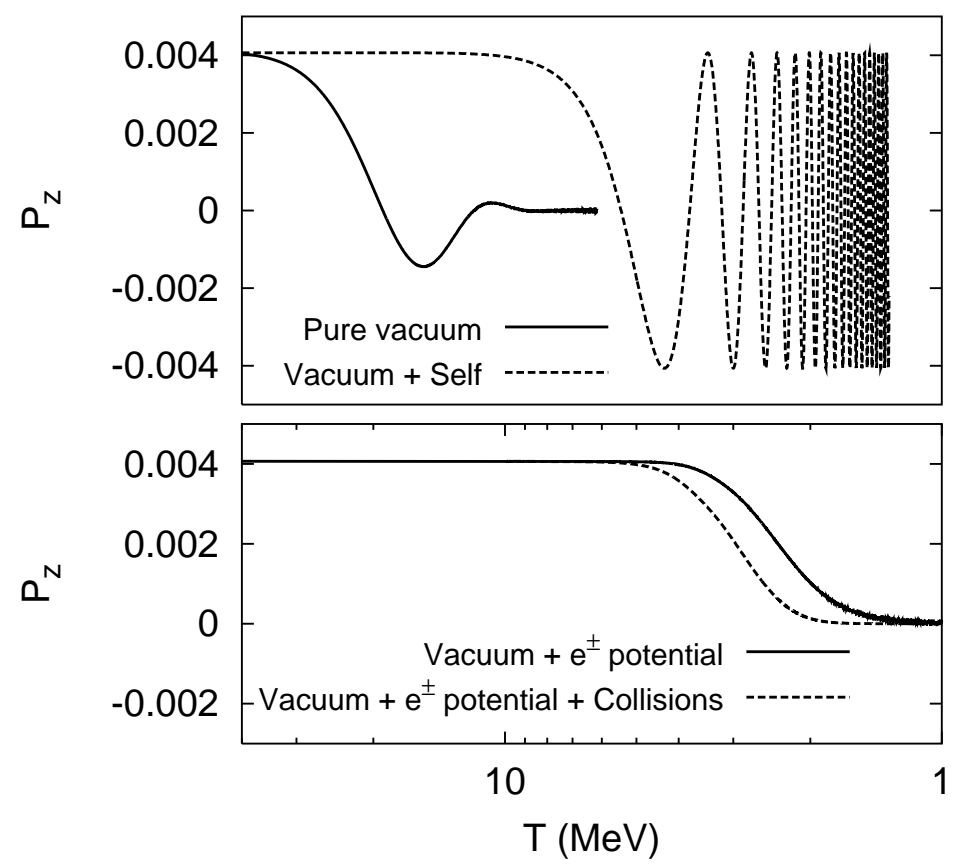

Fig. 1. Evolution of the $z$-component of the integrated polarization vector $\mathbf{P}$ for different situations as explained in the text.

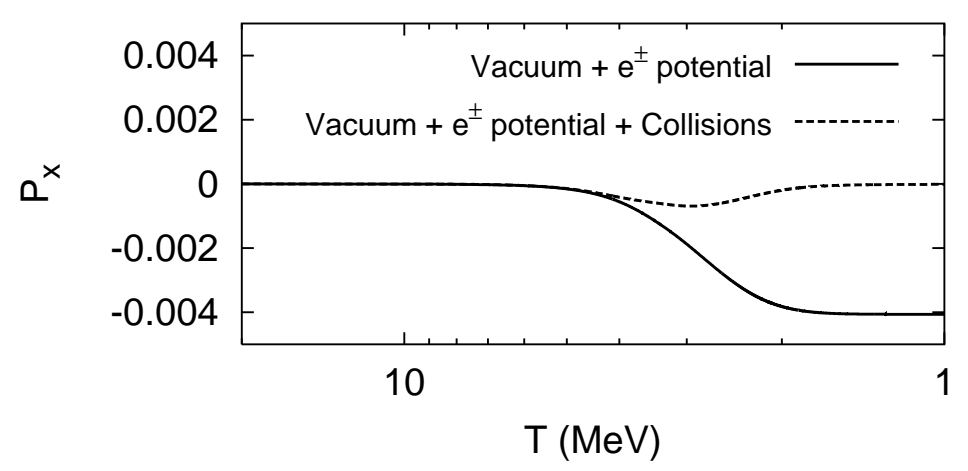

Fig. 2. Evolution of the $x$-component of the integrated polarization vector $\mathbf{P}$ for the same cases as in the lower panel of Fig. 1. 


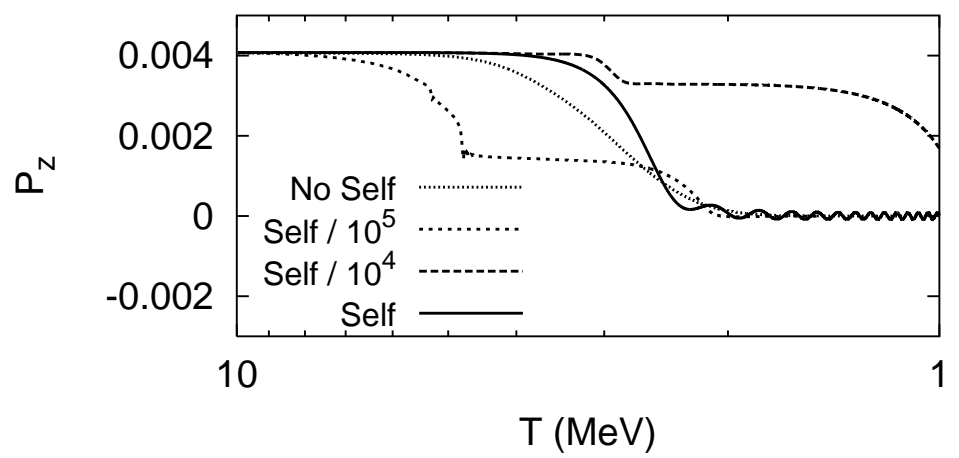

Fig. 3. Evolution of the $z$-component of the integrated polarization vector $\mathbf{P}$ in the presence of the vacuum term, the $e^{+} e^{-}$potential, collisional damping, and varying strength of the neutrino self-interaction.

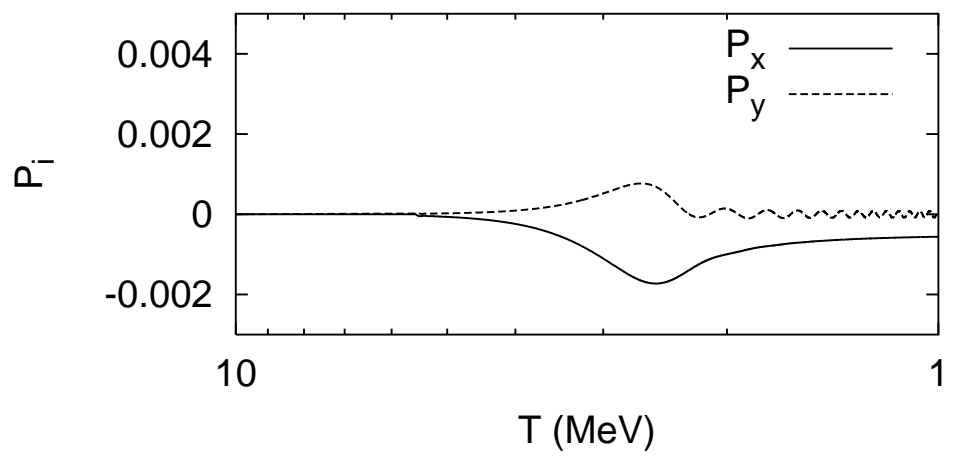

Fig. 4. Evolution of the $x$ and $y$ components of the integrated polarization vector $\mathbf{P}$ in the presence of all effects and full-strength neutrino self-interactions, corresponding to the solid line in Fig. 3. 


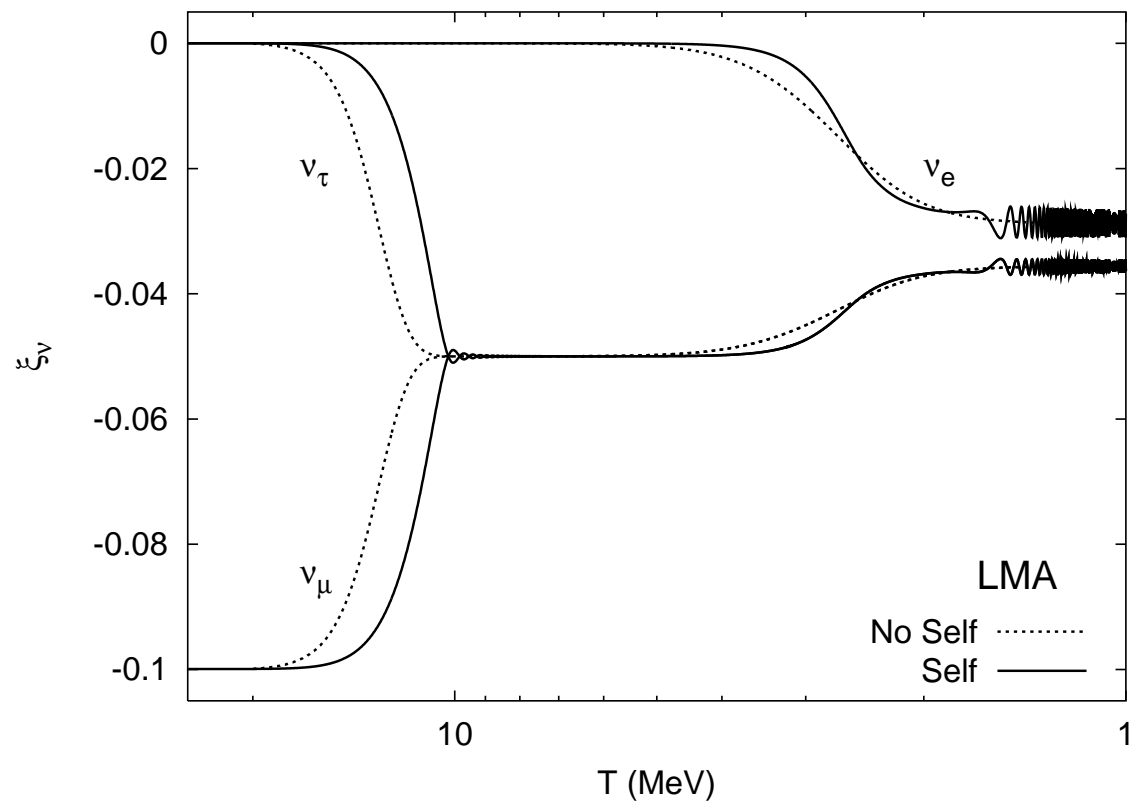

Fig. 5. Evolution of the neutrino degeneracy parameters for the LMA case, $\theta_{13}=0$, and the initial values $\xi_{e}=\xi_{\tau}=0$ and $\xi_{\mu}=-0.1$.

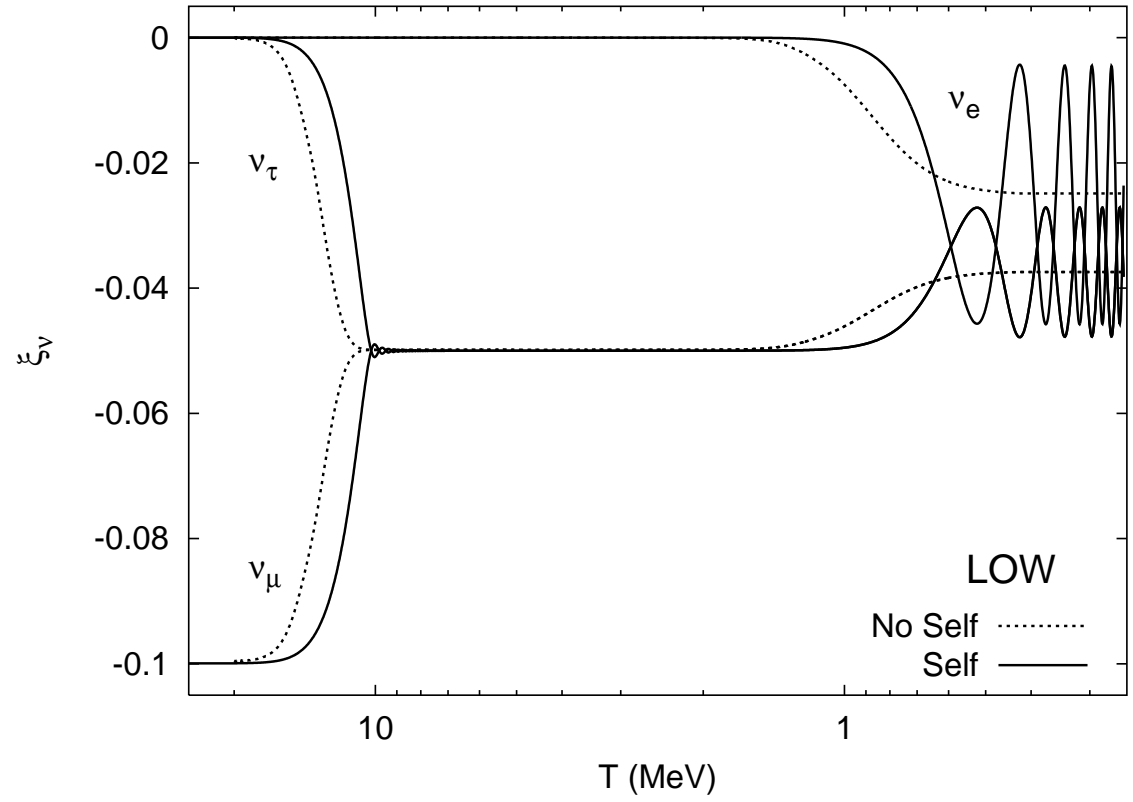

Fig. 6. Evolution of the neutrino degeneracy parameters for the LOW case, $\theta_{13}=0$, and the initial values $\xi_{e}=\xi_{\tau}=0$ and $\xi_{\mu}=-0.1$. 


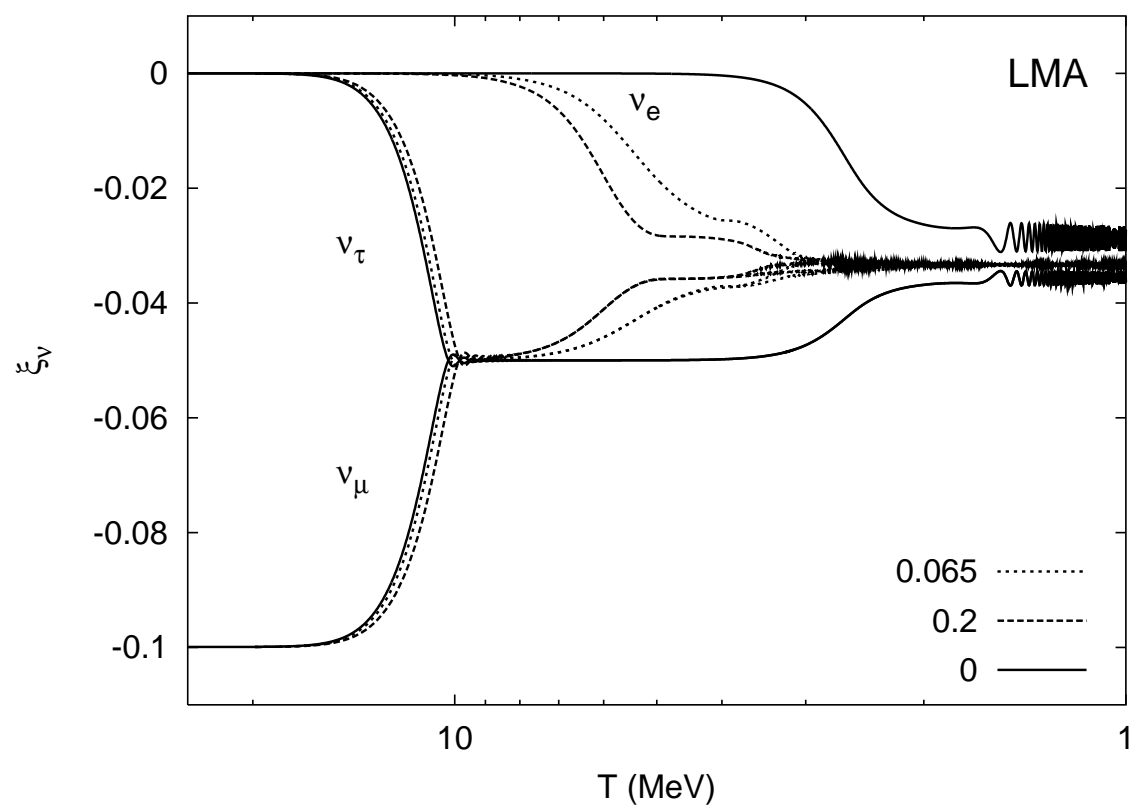

Fig. 7. Evolution of the neutrino degeneracies for different values of $\tan ^{2} \theta_{13}$ in the LMA case for the initial conditions $\xi_{e}=\xi_{\tau}=0$ and $\xi_{\mu}=-0.1$.

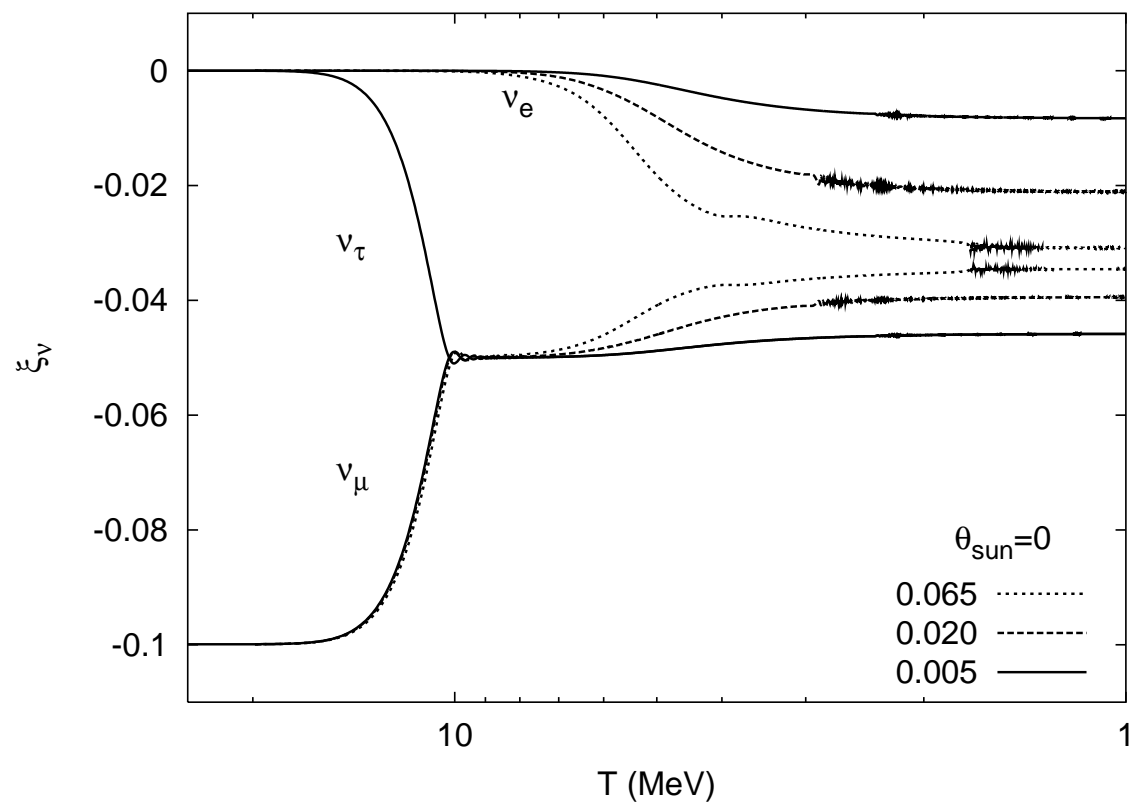

Fig. 8. Evolution of the neutrino degeneracies for different values of $\tan ^{2} \theta_{13}$ in the limit $\Delta m_{\text {sun }}^{2} \simeq 0$ and $\theta_{\text {sun }} \simeq 0$ for the initial conditions $\xi_{e}=\xi_{\tau}=0$ and $\xi_{\mu}=-0.1$. 


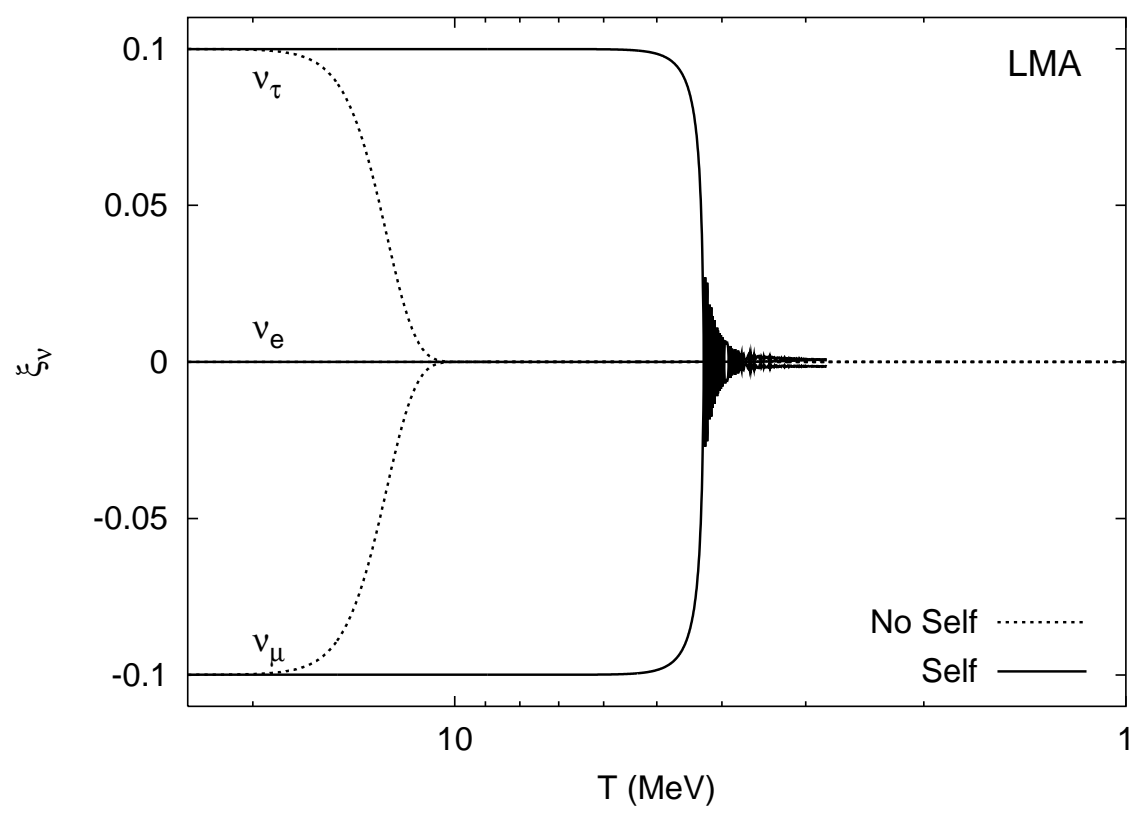

Fig. 9. Evolution of the neutrino degeneracies if initially $\xi_{e}=0$ and $\xi_{\tau}=-\xi_{\mu}=0.1$, with or without neutrino self-interactions.

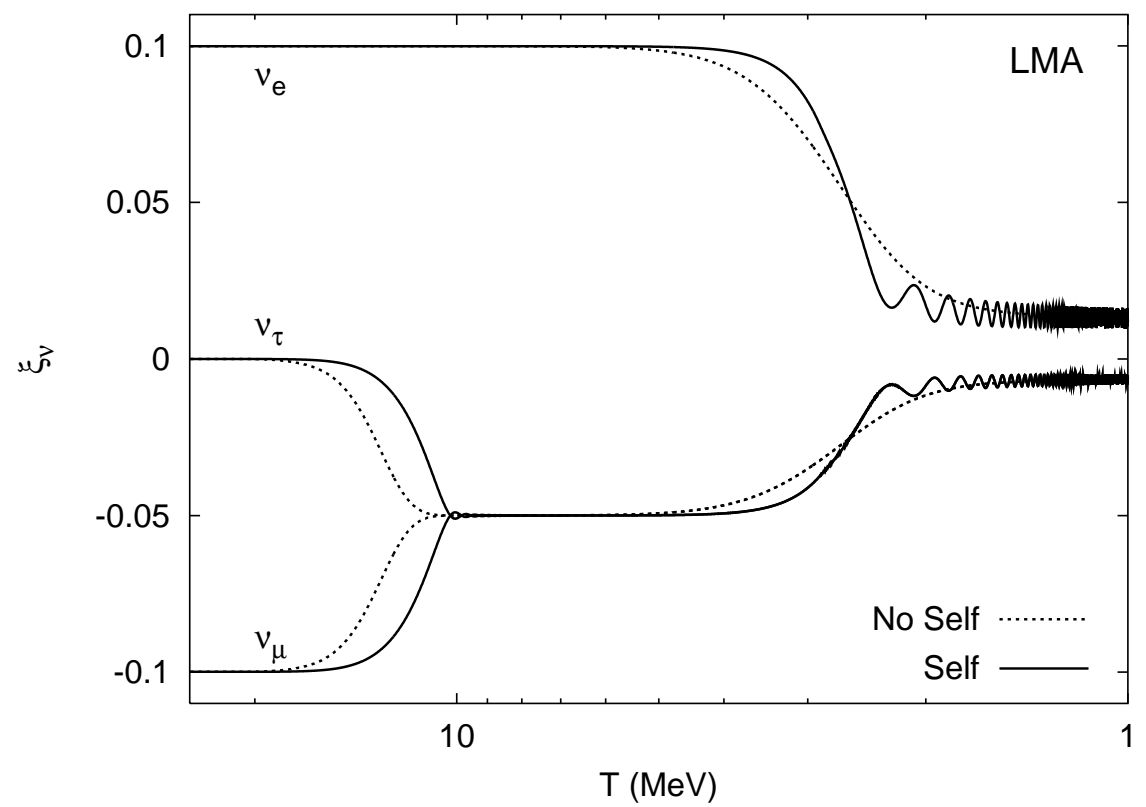

Fig. 10. Evolution of the neutrino degeneracies for the LMA case with $\theta_{13}=0$ with initial conditions $\xi_{\tau}=0$ and $\xi_{e}=-\xi_{\mu}=0.1$, with or without neutrino self-interactions. 